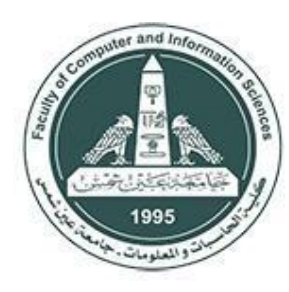

International Journal of Intelligent Computing and Information Sciences

https://ijicis.journals.ekb.eg/

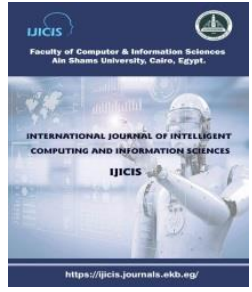

\title{
EARLY DIAGNOSIS OF ALZHEIMER'S DISEASE USING UNSUPERVISED CLUSTERING
}

\author{
Yasmeen Farouk \\ Sherine Rady \\ Department of Information Systems, \\ Department of Information Systems, \\ Faculty of Computer and Information Sciences, \\ Ain Shams University, Cairo, Egypt. \\ yasmin.farouk@cis.asu.edu.eg \\ Faculty of Computer and Information Sciences, \\ Ain Shams University, Cairo, Egypt. \\ srady@cis.asu.edu.eg
}

\begin{abstract}
Alzheimer's disease (AD) is a progressive brain disorder and a very common form of dementia. Neuroimaging techniques, such as Magnetic Resonance Imaging (MRI), produce detailed 3dimensional images of the brain showing insights for amyloid deposits and inflammatory alterations as disease markers. The early diagnosis of AD using MRI provides a good chance for patients to prevent further brain deterioration by stopping the loss of nerve cells. This paper explores the use of unsupervised clustering approaches for the early diagnosis of AD. Though it is very common to use classification techniques for identifying medical diseases, the lack or the inaccuracies of labeled data can generate a problem. In this work, the k-means and k-medoids are compared while employing the Voxel Based Morphometry (VBM) features extracted from the MRI images. The effect of choosing certain local regions of interest (ROIs) for the analysis is also compared to the global whole-brain analysis. The results show that the proposed approach can perform an early diagnosis of $A D$ with an accuracy of $76 \%$.
\end{abstract}

Keywords: Unsupervised Learning, Clustering, K-means, K-medoids, Regions of Interest (ROI), Alzheimer's disease, Magnetic Resonance Imaging (MRI).

\section{Introduction}

In 2018, fifty million people worldwide are reported living with dementia. This number is expected to reach 152 million people by 2050 [1]. About $68 \%$ of this increase, is believed to belong to low- and middle-income countries such as Egypt [2]. Alzheimer's disease (AD) is a progressive brain disorder

* Corresponding author: Yasmeen Farouk

Department of Information Systems, Faculty of Computer and Information Sciences, Ain Shams University, Cairo, Egypt

E-mail address: yasmin.farouk@cis.asu.edu.eg 
and a very common form of dementia. Symptoms appear in the form of memory loss, poor language, irrational problem-solving skills, and the symptoms gradually increase. Patients will eventually be unable to do simple self-management tasks. Although age growth is one of AD's greatest risk factors, it is not considered a normal stage of aging. In the pre-clinical stage of $\mathrm{AD}$, far before symptoms appear, serious brain changes occur by two proteins; beta-amyloid and tau protein. Beta-amyloid protein forms plaques disturbing cell functionality and Tau protein forms tangles blocking cell communication [1]. The two proteins reach abnormal levels in cells of AD brain patient, neurons start to die and the brain starts to shrink.

Unfortunately, AD has no cure. Only progression of the disease might be slowed, and symptoms are treated to raise the comfort and wellbeing of the patient and his family members. However, neuroimaging techniques such as Magnetic Resonance Imaging (MRI), Computed Tomography (CT), and Positron Emission Tomography (PET) produce detailed 3-dimensional images of the brain that show insights for amyloid deposits and inflammatory alterations [1]. MRI describes the integrity of the structures of gray matter (GM) and white matter (WM) in the brain that plays a significant part in the diagnosis of AD. Morphometric methods, such as Voxel-Based Morphometry (VBM) [3], allows the automatic evaluation of GM structures of the suspected AD patients to be compared to the Normal Controls (NC) of aged seniors.

The analysis of neuroimaging techniques can be focused to extract features from certain brain regions or the whole-brain. Studies show that neuro-degeneration in AD emerges from the medial temporal lobe, starting at the entorhinal cortex and progresses to the hippocampus [5]. On the contrary to the wholebrain based approach, the ROI-based approach focuses on one of the four major lobes; the medial temporal lobe. The ROI-based approach registers MRI images to a template of pre-defined brain regions. Although the whole-brain based approach captures the whole pattern of AD by analyzing every part of the brain, practically it suffers from performance issues. The curse of dimensionality caused by the high-dimensional nature of the data in MRI reduces the accuracy and increases the run time. Prespecifying a set of ROIs for processing rather than the whole-brain is an advantageous approach. It poses a good chance for removing noise from data resulting in better disease identification and overall run time reduction.

The high dimensional data encountered in MRI studies for the AD identification problem invited several machine learning approaches to deal with this complex nature. Supervised machine learning uses labeled datasets for training. The underlying learning process analyzes the dataset and builds a function that can be used in the identification. Supervised learning can be viewed as a general term for the classification technique [4]. However, the labeled data used in the training process requires the intervention of a domain expert for the labeling process. The efficiency of the classifiers highly depends on the quality of the labeling process which may be erroneous and time-consuming. On the other hand, unsupervised learning uses an unlabeled dataset. The learning process depends on modeling the structure and finding patterns within the data. Unsupervised learning can be viewed as a general term for the clustering technique. Clustering is not considered an alternative for classification. Despite the low accuracy that clustering techniques may normally achieve compared to classification techniques, it has the power that it is able to discover early key patterns in the data. The advantage of using clustering lies in the automated preliminary insight that the process can provide without any expert intervention. This can give insights for the early diagnosis of diseases at lower costs. 
This paper studies the use of unsupervised clustering approaches, k-means and k-medoids, for the early diagnosis of AD applied on VBM features extracted from MRI. the effect of choosing certain local regions of interest ROIs for analysis compared to the global whole-brain analysis is also introduced and studied.

The rest of this paper is arranged as follows; the related work presented in the literature for the identification of $\mathrm{AD}$ is discussed in Section 2, focusing on the clustering approaches for disease identification. Section 3 describes the scientific approaches and methods used in this study. Section 4 presents the data encountered in the study and explores the experimental results obtained. Finally, Section 5 concludes the findings and results of the paper.

\section{Related Work}

Several supervised and unsupervised learning techniques are proposed in the literature to classify AD patients from NC. Unsupervised clustering techniques proved robustness in identifying clusters of homogeneous AD patients. Hany et al. in [24] studied several clustering techniques and discussed their ability to help in the treatment of AD patients. The study found that clustering techniques can provide useful discriminating features to detect the conversion from early to advanced stages in AD. Escudero et al. in [25] proposed the development of a bioprofile that includes key patterns for the diagnosis of certain diseases in the patients' biodata. The research used k-means clustering technique on ADNI dataset and succeeded to differentiate between two groups pathologic/non-pathologic with an accuracy of $69 \%$. The results of Escudero et al. study are compared to the results obtained in this paper by the proposed clustering approach. G. Tosto et al. in [26] searched for clusters of extra pyramidal signs progression and its development over time to diagnose AD. The study is applied on the data of more than three thousand AD patients from NACC database. It used k-means and was able to identify three clusters of various EPS burden degrees.

Many studies used clustering for an early diagnosis. D. Kar and S. Halder combined unsupervised kmeans clustering with supervised classification for the early detection of brain tumor [36]. Their noninvasive technique analyzed liquid biopsy and used k-means for image processing of CSF. They used Convolutional Neural Network (CNN) and Support Vector Machine (SVM) for tumor classification. Çiklaçandir et al. used k-means for the early detection of breast cancer in [15]. The study proposed a system for identifying the lesion in the breast. K-means-mode was applied by Paul and Hoque in [27] to predict the likelihood of diseases. They tested their approach on medical dataset of diabetes and achieved 95\% accuracy. Gamberger, D. et al. in [28] used multi-layer clustering to identify subpopulations of patients that have homogeneous clinical and biological markers. They concluded that viewing the gender of the suspected patient as a significant descriptor could lead to better effectiveness in the treatment process. The hierarchical agglomerative clustering is another type of clustering technique and is used in several studies. In [29], Y. Noh et al. applied hierarchical agglomerative clustering on MRI of early-stage AD patients to measure the cortical thickness. Their research found three clusters of AD patients with different and distinct clinical features. In [30], A. Cappa et al. used hierarchical agglomerative clustering to identify the neuropathological changes of AD. 
Supervised classification techniques such as SVM proved reliability and robustness in AD/MCI classification as well. Researchers used SVM alone [12] and with other techniques. A. Demirhan in [13] used NN, KNN, and SVM to differentiate between AD and its mild form from NC. The study concluded that SVM was the best classifier among the three with an achieved accuracy reaching $82 \%$. The Open Access Series of Imaging Studies (OASIS) database was used in the study. k. Hackmack et al. used wavelet transform in [14] and proposed a multivariate analysis of sMRI. The study created a feature set composed of scale, directionality, and local information extracted using dual-tree complex wavelet transform.

Deep Learning has a tremendous improvement effect on different science areas over the past few years. Several works have been made to identify AD patients using deep neural network architectures. CNN was widely used for classifying $\mathrm{AD}$ and its prodromal stages. Weiming et al. in [22] designed CNN to predict mild cognitive impairment (MCI) to AD conversion using MRI. Islam et al. in [23] presented CNN for identifying different stages of AD by analyzing MRI data. They identified four AD classes; non-demented, very mild, mild, and moderate AD.

For evaluating the discriminating power of different ROIs over the whole-brain approach, R. Cuingnet et al. in [11] compared ten methods and succeeded to identify between NC, AD, MCI converters, and MCI non-converters. The study included three different types of analysis approaches; five voxel-based, three vertex-based, and two ROI-based. The voxel based methods used the whole-brain for the VBM analysis. The vertex-based methods used the cortical thickness region whereas the two ROI-based approaches used the hippocampus region in the analysis.

The majority of the analytical approaches that are proposed in the literature use features extracted from MRI and other neuroimaging modalities such as CT. VBM approach has been extensively used in the identification of AD using various classification techniques [8-9]. D. Schmitter et al. compared volumebased morphometry approach and the whole-brain VBM approach for AD/MCI classification in [10]. The study in [7] applied volumetric measurements of ROI; where a combined approach of wrapped classification and image segmentation is presented for determining the ROIs from MRI.

Neuroimaging techniques have helped scientists in their research either when each technique is used separately or by combining their strengths. The work presented by [34] combines three neuroimaging techniques; CSF biomarkers, FDG-PET, and MRI. In this study, Zhang et al. classified AD, MCI, and NC using a kernel combination. The study made by Qing Li et al. also used multi-neuroimaging modalities including FDG, sMRI, florbetapir-PET, and PET to classify AD, cognitively unimpaired, and MCI [35]. Their study used discriminant dictionary learning.

\section{Unsupervised Clustering Approach for AD Diagnosis}

The proposed framework of the unsupervised clustering approach used for AD diagnosis is initially supplied with MRI dataset. The Dataset undergoes a pre-processing stage to adjust its flipping, MNI alignment, and ACC positioning. The pre-processed images are supplied to the VBM analysis block where morphometric features are extracted. For exploring the significance of different anatomical ROIs, a masking process is applied on the obtained feature vector where certain ROIs are extracted for further analysis and compared to the whole-brain features.

Images are finally clustered using the ROI or the whole-brain feature vector into two clusters: $\mathrm{AD}$ patient or NC. The block diagram of the framework is illustrated in Figure 1. 


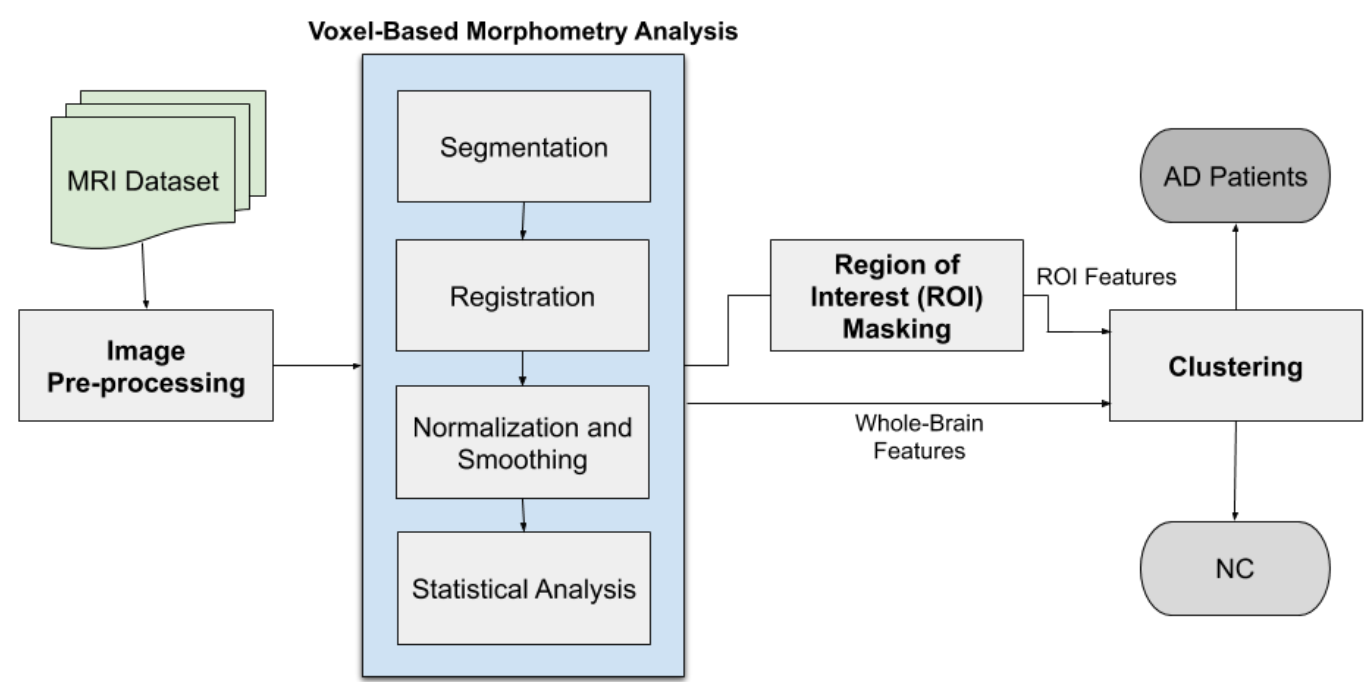

\subsection{MRI Pre-Processing}

Figure 1: Block diagram of Unsupervised Clustering for AD Diagnosis

Before applying VBM analysis, MRI dataset is pre-processed to ensure the images are in a suitable form for processing. Images need to be flipped in the right direction and aligned with Montreal Neurological Institute (MNI) standard space. MNI formed a new standard template by aligning a large number of brain series scans of young healthy normal subjects. MRI dataset is aligned to MNI space by automatically aligning them to this template.

The Anterior Cingulate Cortex (ACC) in all images has to be positioned close to the center of the MRI image i.e. $(0,0,0)$. Unfortunately, this step is done manually for all the images in the dataset. Figure 2 illustrates an MRI with a centered ACC. The MRI images have to be in the NIfTI file format. This format is widely used in imaging informatics for neuroscience. The MRI image in this format contains metadata and voxels that can be in any dimension up to seven dimensions. MRI images used in this work are in three dimensions.

\subsection{Voxel-Based Morphometry Analysis}

VBM examines the primary variations in the structure of the brain. It can be applied to any anatomical scale such as GM, WM, or CSF density. It can effectively measure anatomical atrophy and its expansion. VBM of MRI data involves four processes: segmenting images where GM, WM, and CSF images are extracted, registering the segmented images to estimate the deformations that best align them together, performing spatial normalization to the registered images to the same stereotactic space and then smoothing these normalized images, and finally localizing the variations in the smoothed images by applying a statistical analysis [31]. The statistical analysis output is a parametric map highlighting regions in the image where GM concentration significantly varies between sets. Details of VBM implementation are found in [33]. 


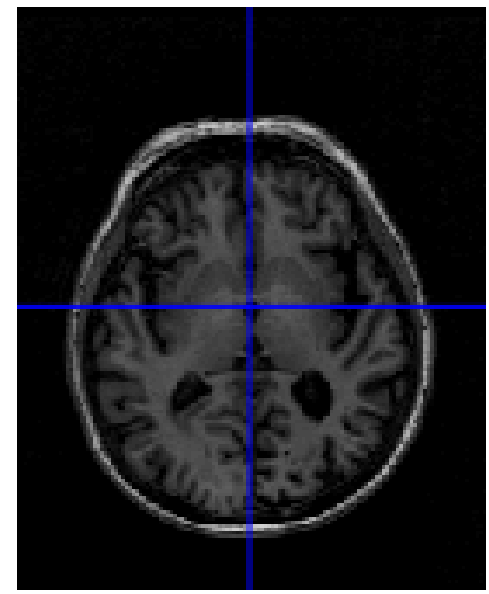

(a)

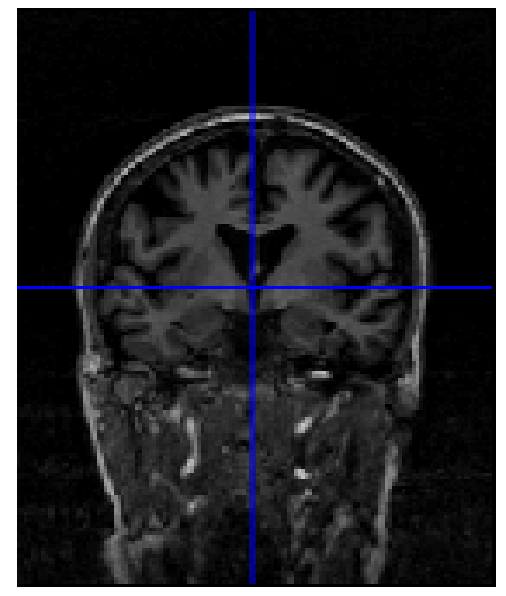

(b)

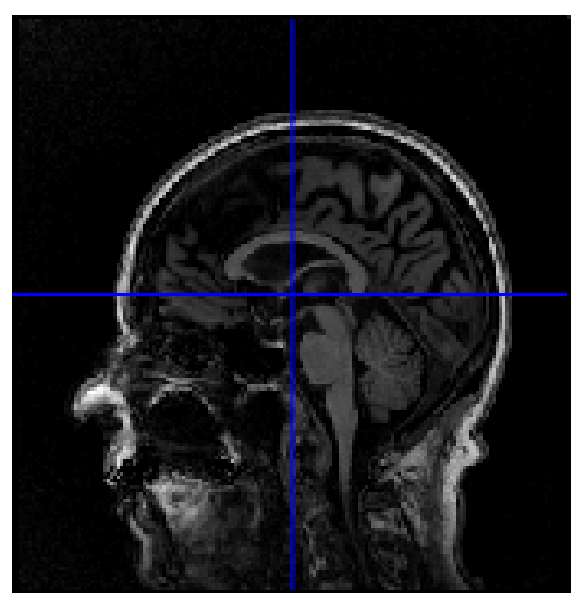

(c)

Figure 2: MRI Centered at Anterior Cingulate Cortex Position: (a) Axial, (b) Coronal, and (c) Sagittal view

\subsection{Region of Interest (ROI) Masking}

At the final point in the statistical analysis of the VBM features analysis, the pre-processed MRI dataset undergoes a statistical t-test as shown in Figure 1. The statistical analysis reveals the important regions of the whole-brain in which GM loss varies between AD and NC. Figure 3 illustrates the GM loss in the cerebellum region in patient brain compared to a normal brain. ROI binary masks are applied on the whole-brain to extract ROIs. ROI masking process specified eight regions for extraction: hippocampus, cerebellum left, cerebellum right, medulla, calcarine, pons, occipital lobe, and frontal lobe. Two feature vectors are ready for clustering; the ROI-based feature vector and the whole-brain feature vector.

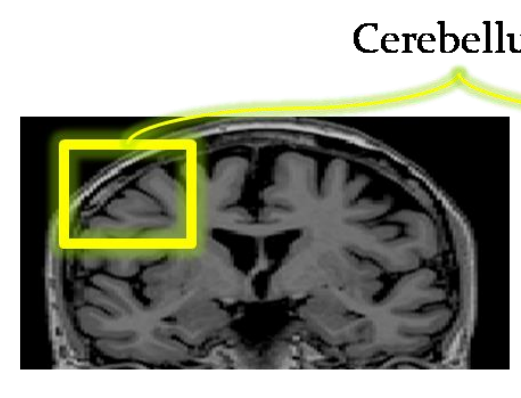

Normal Brain

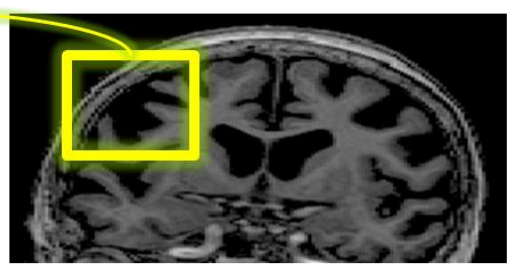

Patient Brain

Figure 3: Cerebellum Region of Interest

\subsection{Unsupervised Clustering Techniques}


Unsupervised learning uses unlabeled dataset. The learning process depends on modeling the structure and finding patterns within the data. Unsupervised learning includes two major types: clustering techniques and association rules. In clustering techniques, the goal is to form clusters where homogeneous data are grouped in, whereas, in association rules, sets of rules are formed that try to describe the data. K-means and k-medoids algorithms are examples of clustering techniques. The proposed framework illustrated in Figure 1 used k-means and k-medoids for clustering.

\subsubsection{K-means}

$\mathrm{K}$-means is a centroid-based partitioning technique. The algorithm uses the centroid of each cluster to represent that cluster. k-means initialize the centroids of $k$ clusters with random items of the dataset. These randomly selected items represent clusters centroids. For each remaining item $i$ in the dataset $D$, the algorithm calculates the Euclidean distance between this item and each cluster centroid $c$. The item is assigned to the cluster of the centroid $c$ of the smallest Euclidean distance. After each iteration, the centroid of each cluster is updated by the mean value of all items belonging to this cluster. All items in all clusters are reassigned after the update to the nearest centroid. The iterations and the update of the centroid continue until all the clusters remain stable. Equation (1) explains the objective function $J$ of k-means algorithm where $k$ is the number of clusters, $n$ is the total number of items in the dataset, $x$ is a dataset item, and $c$ is the cluster centroid. Equation (1) calculates the distance using a Euclidean distance function.

$$
J=\sum_{j=1}^{k} \sum_{i=1}^{n}\left\|x_{i}^{(j)}-c_{j}\right\|^{2}
$$

The algorithm tries to form compact and separate clusters. The items in the dataset are distributed into $\mathrm{k}$ clusters with an objective function that maximizes the similarity between items of the same cluster and minimizes the similarity to other items in other clusters. However, the algorithm remains very sensitive to the outlier items in the dataset. These items are dissimilar from the majority of items in the dataset. Assigning this outlier to a cluster will cause distortion to the mean value of the cluster centroid which in turn affects the assignment of other items to this cluster. K-medoids algorithm proposed a good solution to this problem.

\subsubsection{K-medoids}

The algorithm redefined the centroid of the cluster. Actual items in the dataset are used as the cluster centroid instead of calculating the mean value of all the items in the cluster. The remaining items in the dataset are assigned to the cluster of which they are most similar to its 'actual item' centroid.

In the proposed clustering block illustrated in Figure 1, the cosine distance function is used to compute the distance between dataset items and the centroids. Cosine function is shown in Equation (2) where $d$ is the distance between $X$ and $Y$ items.

$$
d=\frac{X . Y}{\|X\|\|Y\|}
$$

\section{Experiments and Results}




\subsection{Dataset}

Alzheimer's Disease Neuroimaging Initiative (ADNI) database is used in this work [34]. In 2004, ADNI was developed to help in the early diagnosis of AD by providing chemical biomarkers, genetics data, clinical data, PET and MRI image data.

MRI neuroimaging technique suffers from intensity non-uniformity which degrade the quality of the acquired data. The data intensity varies in an irrelevant pattern. The pulse sequence of the acquisition and the radio-frequency coil are both responsible for this problem. The ADNI images have the advantage of being pre-processed for a reduced intensity non-uniformity after applying the N3 histogram peak sharpening algorithm.

The demographic information of MRI studied subjects is shown in Table 1. A total of 275 MRI images are studied, including $113 \mathrm{NC}$ and $162 \mathrm{AD}$ patients. Almost two-third of the NC cases are males while less than half of the AD cases are males. The age mean is almost the same in both groups NC and AD. However, standard deviation of the AD group is higher than the NC group. The Mean value for MiniMental State Examination (MMSE) for both groups is almost similar with a lower standard deviation value for $\mathrm{AD}$ group.

Table 1: Demographics of the Dataset obtained from ADNI

\begin{tabular}{|l|l|l|}
\hline & Normal Control & Alzheimer's Disease Patient \\
\hline & Total Number $=\mathbf{1 1 3}$ & Total Number $=\mathbf{1 6 2}$ \\
\hline Male/female & $72 / 41$ & $71 / 91$ \\
\hline Age: $\boldsymbol{\mu ( \sigma )}$ & $77.49(5.88)$ & $73.82(7.63)$ \\
\hline MMSE: $\boldsymbol{\mu ( \sigma )}$ & $25.74(7.74)$ & $21.54(3.92)$ \\
\hline
\end{tabular}

\subsection{Experimental Work}

The performance tests of the two clustering approaches are conducted on the whole-brain features and the ROIs features as illustrated in Figure 1. Two measures are used to evaluate the clustering quality of k-means and k-medoids, Accuracy and Rand Index. Accuracy is calculated by the formula shown in equation (3) and Rand Index is calculated by the formula shown in equation (4) where TP, TN, P, and N refer to the number of samples representing true positive, true negative, positive, and negative respectively.

$$
\begin{aligned}
& \text { Accuracy }=(\mathrm{TP}+\mathrm{TN}) /(\mathrm{P}+\mathrm{N}) \\
& \text { Rand Index }=(\mathrm{TP}+\mathrm{TN}) /(\mathrm{TP}+\mathrm{TN}+\mathrm{P}+\mathrm{N})
\end{aligned}
$$

\subsubsection{Performance Tests of k-means and k-medoids on Whole-Brain}

Table 2 demonstrates the accuracy, rand Index, and run time results obtained by k-means and k-medoids clustering on the whole-brain features. k-means obtained slightly better results than k-medoids in terms of accuracy and rand Index. However, k-medoids consumed about double the run time of k-means. The 
proposed clustering approach is compared to the results obtained by Escudero et al. in [25]. The achieved accuracy of the proposed approach is better by $7 \%$ than the study in [25].

Table 2: Performance Results of k-means and k-medoids Clustering

\begin{tabular}{|l|l|l|l|}
\hline & k-means & k-medoids & $\begin{array}{l}\text { k-means by Escudero et al. } \\
\text { in [25] }\end{array}$ \\
\hline Accuracy $(\boldsymbol{\%})$ & $\mathbf{7 6 . 3 \%}$ & $75.27 \%$ & $69 \%$ \\
\hline Rand Index & 0.64 & 0.63 & - \\
\hline Run Time (sec) & 43.9 & 82.13 & - \\
\hline
\end{tabular}

\subsubsection{Performance Tests of k-means and k-medoids on ROIs}

Eight brain regions are defined as ROIs. The regions include hippocampus, cerebellum left, cerebellum right, medulla, calcarine, pons, occipital lobe, and frontal lobe and are grouped as follows:

- ROI1 includes the hippocampus region only.

- ROI2 includes cerebellum right, cerebellum left, and the hippocampus.

- ROI3 includes calcarine, cerebellum right, cerebellum left, and the hippocampus.

- ROI4 includes frontal lobe, calcarine, cerebellum right, cerebellum left, and the hippocampus.

- ROI5 includes pons, occipital lobe, medulla, frontal lobe, calcarine, cerebellum right, cerebellum left, and the hippocampus.

The accuracy and run time results of measuring the effect of analyzing these ROIs instead of the wholebrain on k-means are illustrated in Figure 4 and 6 respectively. Figure 5 and 7 illustrate the accuracy and run time results obtained by k-medoids on the same ROIs respectively. The results of analyzing ROI failed to obtain higher accuracy than the whole-brain demonstrated in Table 2. The average accuracy obtained by both clustering approaches on all ROIs is $60 \%$. ROI5 obtained the best accuracy using kmeans and ROI3 obtained the best accuracy using k-medoids. The Figures show the run time increases from ROI1 to ROI5 due to the increase in the feature vector size. However, the measurements of the run time of ROIs remain far less than the measurements obtained by the whole-brain demonstrated in Table 2. The maximum ROI run time obtained by k-means is less than 4 seconds and by k-medoids is less than 7 seconds. The whole-brain approach consumes more than 40 seconds by k-means and more than 80 seconds in k-medoids. Despite the remarkable drop in the accuracy obtained by analyzing ROI, it presents a decrease in the run time by $13 \%$ compared to the whole-brain. 


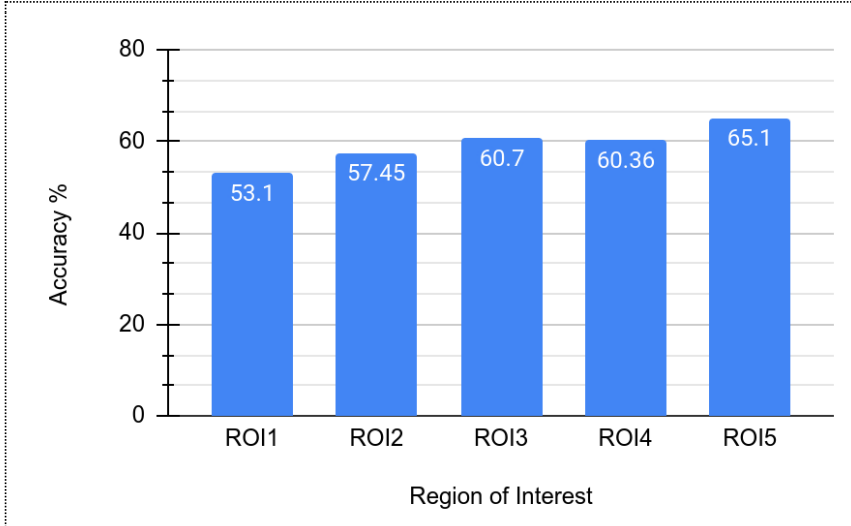

Figure 4: Accuracy of k-means clustering using different

ROIs

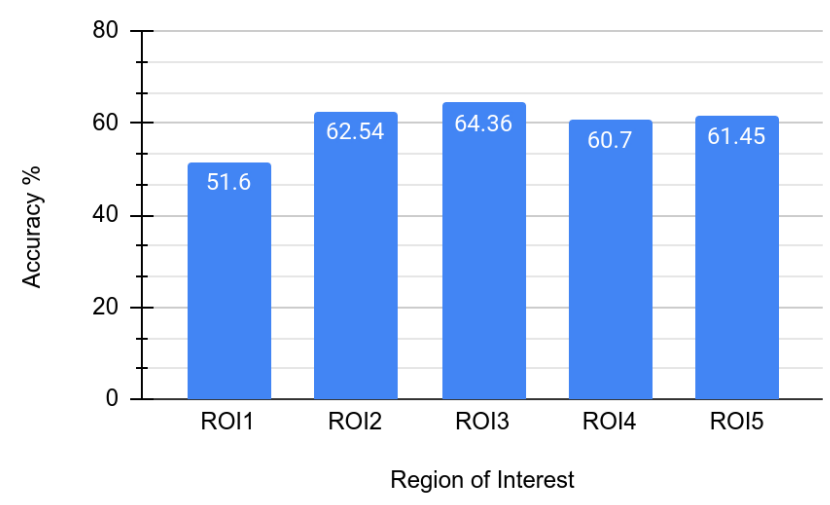

Figure 5: Accuracy of k-medoids clustering using different ROIs

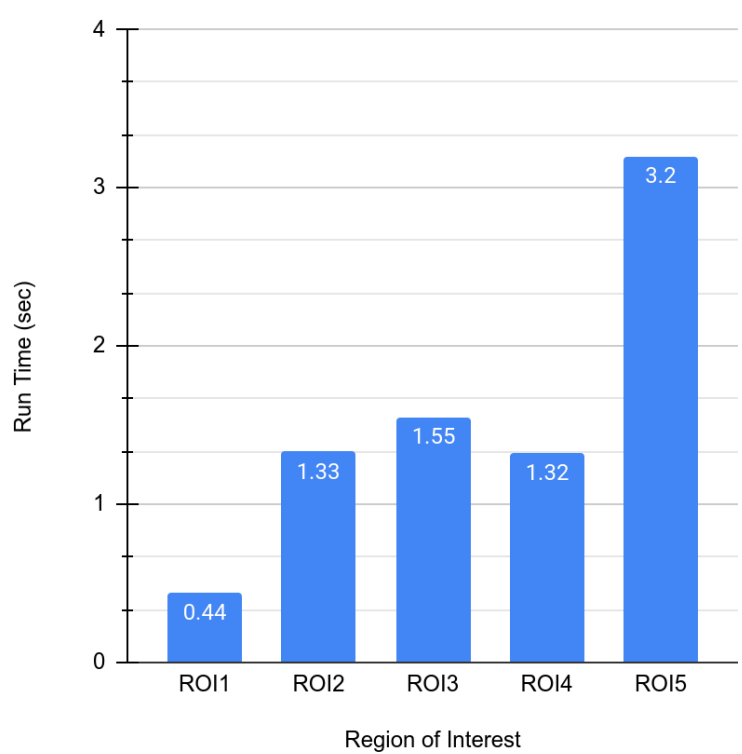

Figure 6: Run Time of k-means clustering for the different

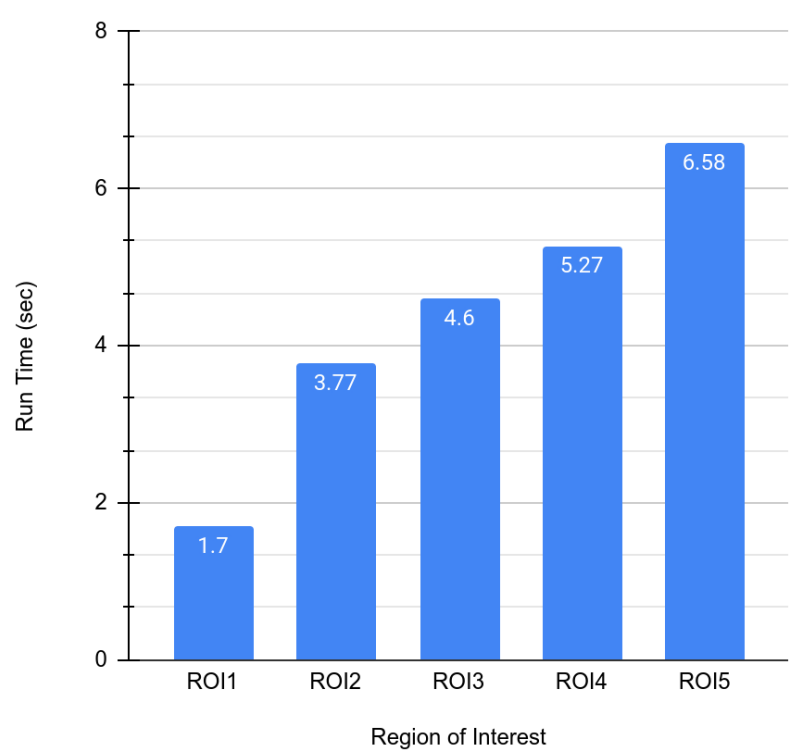

Figure 7: Run Time of k-medoids clustering for the different

ROIs

The work presented in this paper is executed on a 64-bit Intel i5 processor with a memory size of 11.5GB running on Ubuntu 18.04.5.

\subsection{Discussion}

$\mathrm{k}$-medoids is considered more robust than k-means if the data contains noise or outliers. However, the complexity of k-medoid is much higher than k-means. The size of the dataset and the number of clusters used in this study are not large which makes k-means consumes less time than k-medoids. Moreover, 
the nature of the gray image MRI dataset does not contain any outliers which makes applying kmedoids less advantageous than k-means. This explains the obtained results which show that k-means achieved higher accuracy than k-medoids and in shorter run time.

The analysis of the whole-brain features obtained far better results than the analysis of the ROI features. The clustering approach used in the analysis made use of every key pattern in the brain MRI image to discover the intrinsic grouping inside the data. However, the ROI results are still accepted in an early detection diagnosis as it consumed less run time than whole-brain.

The accuracy obtained by classification techniques normally exceeds $80 \%$ in many studies. However, this high accuracy comes at the expense of time and effort used by domain experts to label the training datasets. K-means and k-medoids used in this work were able to achieve only $4 \%$ lower accuracy than other classification techniques suggesting that clustering techniques are able to provide a good reliable preliminary insight for early diagnosis as well as saving the time and effort of domain experts.

\section{Conclusion}

The work presented in this paper explores the advantages of using unsupervised clustering approaches, $\mathrm{k}$-means and k-medoids, for the early diagnosis of $\mathrm{AD}$. The proposed framework applied the clustering approaches on Voxel Based Morphometry features extracted from MRI. The achieved results show that k-means obtained slightly higher accuracy than k-medoids and consumed nearly half of the k-medoids run time. The paper explores also the effect of choosing certain local regions of interest ROIs for analysis compared to the global whole-brain analysis. The whole-brain approach achieved more than $10 \%$ higher accuracy than the ROI approach. However, the run time of the ROI approach is $13 \%$ lower than the whole-brain approach. The proposed approach managed successfully to obtain an early AD diagnosis with an accuracy of $76 \%$. The clustering techniques used in the proposed approach provided an automated preliminary insight discovering early key patterns in the data with reliable accuracy.

\section{References}

1. Patterson, Christina. "World Alzheimer report 2018: the state of the art of dementia research: new frontiers." Alzheimer's Disease International (ADI): London, UK(2018): 32-36.

2. Prince, Martin James. World Alzheimer Report 2015: the global impact of dementia: an analysis of prevalence, incidence, cost and trends. Alzheimer's Disease International, 2015.

3. Mechelli, Andrea, et al. "Voxel-based morphometry of the human brain: methods and applications." Current Medical Imaging 1.2 (2005): 105-113.

4. Han, Jiawei, Jian Pei, and Micheline Kamber. Data mining: concepts and techniques. Elsevier, 2011.

5. Magnin, Benoît, et al. "Support vector machine-based classification of Alzheimer's disease from whole-brain anatomical MRI." Neuroradiology 51.2 (2009): 73-83.

6. Farouk, Yasmeen and Rady, Sherine. "Identifying Alzheimer's disease using feature reduction of GLCM and supervised classification techniques". Neurological Disorders and Imaging Physics, Volume3 Application to autism spectrum disorders and Alzheimer's (2019). Pages 12-1-12-26. 
7. Hidalgo-Muñoz, Antonio R., et al. "Regions of interest computed by SVM wrapped method for Alzheimer's disease examination from segmented MRI." Frontiers in aging neuroscience 6 (2014): 20.

8. Hirata, Yoko, et al. "Voxel-based morphometry to discriminate early Alzheimer's disease from controls." Neuroscience letters 382.3 (2005): 269-274.

9. Chételat, Gaël, et al. "Using voxel-based morphometry to map the structural changes associated with rapid conversion in MCI: a longitudinal MRI study." Neuroimage27.4 (2005): 934-946.

10. Schmitter, Daniel, et al. "An evaluation of volume-based morphometry for prediction of mild cognitive impairment and Alzheimer's disease." NeuroImage: Clinical 7 (2015): 7-17.

11. Cuingnet, Rémi, et al. "Automatic classification of patients with Alzheimer's disease from structural MRI: a comparison of ten methods using the ADNI database." neuroimage 56.2 (2011): 766-781.

12. Ferreira, Luiz K., et al. "Support vector machine-based classification of neuroimages in Alzheimer's disease: direct comparison of FDG-PET, rCBF-SPECT and MRI data acquired from the same individuals." Brazilian Journal of Psychiatry 40.2 (2018): 181-191.

13. Demirhan, Ayşe. "Classification of structural MRI for detecting Alzheimer's disease." International Journal of Intelligent Systems and Applications in Engineering(2016): 195-198.

14. Hackmack, Kerstin, et al. "Multi-scale classification of disease using structural MRI and wavelet transform." Neuroimage 62.1 (2012): 48-58.

15. Çiklaçandir, Fatma Günseli Yaşar, et al. "Lesion Detection from the Ultrasound Images Using KMeans Algorithm." 2019 Medical Technologies Congress (TIPTEKNO). IEEE, 2019.

16. Li, Zhaohua, et al. "Evaluation of different classification methods for the diagnosis of schizophrenia based on functional near-infrared spectroscopy." Journal of Neuroscience Methods 241 (2015): 101110.

17. Beheshti, Iman, Hasan Demirel, and Alzheimer's Disease Neuroimaging Initiative. "Featureranking-based Alzheimer's disease classification from structural MRI." Magnetic resonance imaging 34.3 (2016): 252-263.

18. Zhang, Yudong, et al. "A hybrid method for MRI brain image classification." Expert Systems with Applications38.8 (2011): 10049-10053.

19. Toro, César A. Ortiz, et al. "Supervoxels-based histon as a new alzheimer's disease imaging biomarker." Sensors18.6 (2018): 1752.

20. Herrera, Luis Javier, et al. "Classification of MRI images for Alzheimer's disease detection." 2013 International Conference on Social Computing. IEEE, 2013.

21. Ding, Yiming, et al. "A deep learning model to predict a diagnosis of Alzheimer disease by using 18F-FDG PET of the brain." Radiology 290.2 (2019): 456-464.

22. Lin, Weiming, et al. "Convolutional neural networks-based MRI image analysis for the Alzheimer's disease prediction from mild cognitive impairment." Frontiers in neuroscience12 (2018): 777.

23. Islam, Jyoti, and Yanqing Zhang. "Brain MRI analysis for Alzheimer's disease diagnosis using an ensemble system of deep convolutional neural networks." Brain informatics5.2 (2018): 2.

24. Alashwal, Hany, et al. "The application of unsupervised clustering methods to Alzheimer's Disease." Frontiers in computational neuroscience 13 (2019): 31. 
25. Escudero, Javier, John P. Zajicek, and Emmanuel Ifeachor. "Early detection and characterization of Alzheimer's disease in clinical scenarios using Bioprofile concepts and K-means." 2011 Annual International Conference of the IEEE Engineering in Medicine and Biology Society. IEEE, 2011.

26. Tosto, G., Monsell, S. E., Hawes, S. E., Bruno, G., and Mayeux, R. (2016). Progression of extrapyramidal signs in Alzheimer's disease: clinical and neuropathological correlates. J. Alzheimers Dis. 49, 1085-1093. doi: 10.3233/JAD-150244

27. Paul, R., and Hoque, A. S. M. L. (2010). "Clustering medical data to predict the likelihood of diseases," in Digital Information Management (ICDIM), 2010 Fifth International Conference on (IEEE) (Thunder Bay, ON), 44-9.

28. Gamberger, D., Ženko, B., Mitelpunkt, A., Shachar, N., and Lavrac, N. (2016a). Clusters of male and female Alzheimer's disease patients in the Alzheimer's disease neuroimaging initiative (ADNI) database. Brain Inform. 3, 169-179. doi: 10.1007/s40708-016-0035-5.

29. Noh, Y., Jeon, S., Lee, J. M., Seo, S. W., Kim, G. H., Cho, H., et al. (2014). Anatomical heterogeneity of Alzheimer's disease based on cortical thickness on MRIs. Neurology 83, 19361944.

30. Cappa, A., Ciccarelli, N., Baldonero, E., Martelli, M., and Silveri, M. C. (2014). Posterior ad-type pathology: cognitive subtypes emerging from a cluster analysis. Behav. Neurol. 2014:259358.

31. Ashburner, John, and Karl J. Friston. "Voxel-based morphometry — the methods." Neuroimage 11.6 (2000): 805-821.

32. Jolliffe, Ian T. "Principal component analysis: a beginner's guide-I. Introduction and application." Weather 45.10 (1990): 375-382.

33. Farouk, Yasmeen, Sherine Rady, and Hossam Faheem. "Statistical features and voxel-based morphometry for alzheimer's disease classification." 2018 9th International Conference on Information and Communication Systems (ICICS). IEEE, 2018.

34. Zhang, Daoqiang, et al. "Multimodal classification of Alzheimer's disease and mild cognitive impairment." Neuroimage 55.3 (2011): 856-867.

35. Li, Qing, et al. "Classification of Alzheimer's disease, mild cognitive impairment, and cognitively unimpaired individuals using multi-feature kernel discriminant dictionary learning." Frontiers in computational neuroscience 11 (2018): 117.

36. Kar, Deblina, and Sourav Halder. "Early Detection of Brain Tumor by Using K-Means Clustering, Convolutional Neural Network and Support Vector Machine without any Imaging Test." 2019 International Conference on Information Technology (ICIT). IEEE, 2019. 